\title{
Adiabatic Thermodyhamics
}

November 16, 2018

\author{
Christian Fronsdal
}

Dept. of Physics and Astronomy, university of California Los Angeles USA

\begin{abstract}
$A B S T R A C T$ This is a review of efforts to discover action principles for Hydrodynamics and Thermodynamics. The work began with a determined attempt to create sources for Einstein's equations that describe continuous matter - as opposed to point particles. This requires a relativistic action principle. This report is mainly about (non relativistic) Adiabatic Thermodynamics, non equilibrium thermodynamics based on the method of Gibbs. The main relations of classical thermodynamics can be derived from an action principle, in the classical global form and in the local form advocated by Callen. The entropy is defined as the negative derivative of the free energy (free energy density) with respect to the volume (density). This is integrated with an action principle for hydrodynamics that was known to Lagrange in 1760. It is strongly limited to irrotational velocity fields, nevertheless it provides the needed Lagrangian for a large part of thermodynamics. This is the subject of the first 3 sections. It includes a brief review of a new theory of mixtures.

The most difficult part of the program has been the discovery of Conservative Hydrodynamics with the required four independent degrees of freedom, with general flows. We give an account of the genesis and the structure of this theory, then a tour of recent applications.
\end{abstract}




\section{Introduction}

\section{Minimal energy and maximal entropy.}

The two ideas in the title are among the most basic concepts of theoretical physics. The central point that was made by Gibbs in his path breaking paper (1878) was that, in thermodynamics, the two are equivalent: maximum entropy implies minimum energy and vice versa.

This sheds light on both principles. Either one can be elevated to an axiom: then the other becomes a theorem; they cannot both be axioms and they are not independent theorems. The choice recommended by Callen (1960), and widely accepted, is to take entropy as the more basic concept. But it can perhaps be argued that there must be a more elevated standpoint from which the dominant fact is the equivalence of the two statements. This appears to have been the attitude of Gibbs (1878); his verbal argument can be illustrated as in Fig. 1, where the abscissa is the value of the entropy and the ordinate is the energy. Gibbs argues that when the entropy can not be increased then the energy cannot be decreased. This amounts to drawing a line with positive slope in the $E, S$ plane and declaring that allowed configurations are the points the left of the line.

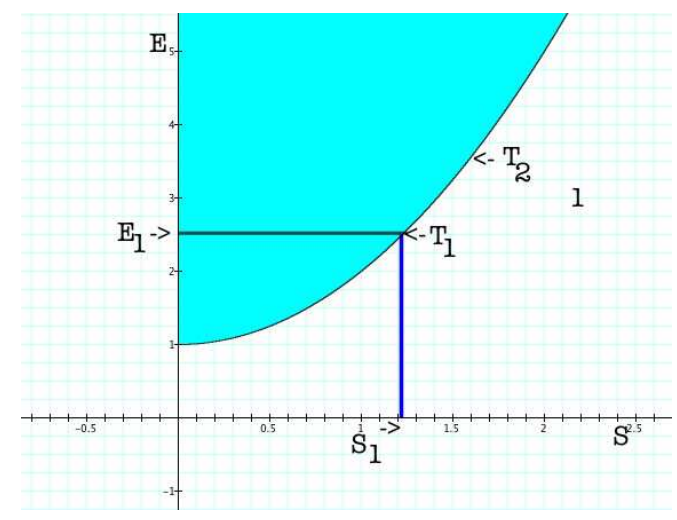

Fig 1. Illustration of Gibbs' equivalence theorem. Physical configurations are in the shaded region. Gibbs shows that the limiting curve has positive slope.

While Gibbs' proof was almost entirely verbal, it is easy to reduce it to formal statements expressed by equations. It will be instructive to do so.

Consider a simple system with variables $V, T, S, P$ and the function

$$
A_{1}(V, T, S, P)=F(V, T)+S T+V P .
$$

With Gibbs, we fix the values of $S$ and $P$ and postulate that this function is extremal with respect to arbitrary variations of $V$ and $T$; this leads to the Euler-Lagrange equations

$$
\frac{\partial F}{\partial V}+P=0, \quad \frac{\partial F}{\partial T}+S=0
$$

These are the basic relations of thermodynamics and these are the equations used by Gibbs to prove the equivalence of 'Maximum entropy' and 'Minimum energy'. 
The second equation, "the adiabatic condition", is an algebraic equation for the temperature. It can be used to express $T$ in terms of $V$ and $S$; projecting thermodynamics on hydrodynamics, parametrized by the value of the entropy.

We shall limit the discussion to configurations in which the volume is fixed. Thermodynamics is thus an action principle and the action is $F+S T$. Gibbs does not characterize the energy, nor the entropy, instead he uses the relations that define thermodynamics to prove the equivalence of the two statements. In practice, the existence of a configuration with minimal energy is an axiom that is used to select appropriate expressions for the function $F(V, T)$; then $S$ is defined by the second condition in (1.2). This point of view is complimentary to that of Callen; it is not in opposition to it.

\section{The discovery and the definition of entropy}

There were three important milestones.

1. Kelvin noticed that the gas law takes the form

$$
p=\mathcal{R} \rho\left(T_{C}-273\right)
$$

and defined the absolute temperature as $T=T_{C}-273$.

2. Clausius (1859) discovered the role of $1 / T$ as the multiplier that makes the differential

$$
d S=\frac{d Q}{T}
$$

exact and proposed the name "entropy" for the state variable $S$.

3. Laplace (see Biot 1802) used the concept of adiabatic changes $(d S=0)$ to obtain an accurate prediction of the speed of sound in air. Note that he makes use of the entropy in an application that is out of equilibrium.

In this context but in the idiom developed later, the new variable $S$ was defined as a derivative of the free energy:

$$
S=-\left.\frac{\partial F}{\partial T}\right|_{V} .
$$

This definition has survived; it is valid for equilibria and for all other configurations. The problem of how to define the entropy for configurations out of equilibrium is not a problem for thermodynamics. But in 1868 Boltzmann created statistical mechanics and, with stunning insight proposed a new type of entropy. An object of statistical mechanics, it turned out to be identifiable with the entropy of equilibrium thermodynamics. Boltzmann's definition applies to statistical equilibria, till now it has not been generalized and that is an important problem for statistical mechanics, but not for thermodynamics. The proof is in the fact that Gibbs' use of entropy, and Laplace's early application of it, both apply to non-equilibrium configurations.

This is more than a question of semantics, for the question has lain as a damper over thermodynamics. We shall see that adopting the Gibbs-Laplace attitude to entropy has wide implications.

Certainly, entropy as a concept is more elusive than energy; for this reason it is natural to take the energy as more basic. We may not have a completely satisfactory 
definition of 'energy' in general, but we have done quite well for many particular systems. In thermodynamics, armed with Gibbs' statement about the equivalence between minimal energy and maximal entropy, this is a already big step towards a definition of the entropy. The function $F$ can be identified with the Hamiltonian and then (2.1) is a cogent definition of the entropy.

The problem is not to define the entropy but to determine the action.

\section{The thermodynamic action}

Gibbs begins with a discussion of a simple system where the variables are global and the configurations are labelled by 4 real variables, then goes on to make applications of a much greater complexity by applying acute physical insight. In a first step, later formalized as Callen's 'main assumption' (Callen 1960), one assumes that the fundamental relations are satisfied pointwise. The formula for the action needs an extra term, as in

$$
\int d^{3} x\left( \pm \rho \vec{v}^{2} / 2+f(\rho, T)+s T+P\right), \ldots ?,
$$

where $f$ is the density of free energy and the entropy density $s$ is a linear function of the mass density,

$$
s=\rho S .
$$

In elementary applications the specific entropy density $S$ is a constant.

To go on we invoke Lagrange's action principle for irrotional hydrodynamics, with

$$
A_{2}=\int d^{3} x\left(\rho\left(\dot{\Phi}-\vec{v}^{2} / 2\right)-W[\rho]\right)
$$

Here the velocity field is the negative gradient of a velocity potential,

$$
\vec{v}=-\vec{\nabla} \Phi
$$

The term $\vec{v}^{2} / 2$ is part of the energy density and $\dot{\Phi}$ is the "kinetic" term, the part of the Lagrangian with time derivatives. Uniting the actions (1.2) and (1.3) one arrives at the action for irrotational thermodynamics,

$$
A=\int d^{3} x d t\left(\rho\left(\dot{\Phi}-\vec{v}^{2} / 2\right)-f-s T\right)
$$

A large part of thermodynamics is concerned with static configurations of systems with a single components. The action (3.3) allows to consider motions, but only under the severe limitation to irrotational velocity fields.

\section{The value of an action principle in physics}

The most highly developed branch of theoretical physics is high energy Particle Physics. The amazing power of prediction of Electromagnetism, Quantum Mechanics and Quantum Field Theory, successfully integrated to form the core of high energy physics, 
is without parallel. Outstanding examples are theoretical predictions of fundamental constants to an accuracy of 8-10 significant figures and the prediction of the existence of elementary particles, with the values of their masses, before their actual experimental discovery. Two circumstances helped to bring this about. Perhaps most of all, the driving force has been the insistence on logical consistency; as in the case of renormalizability of quantum field theories, an obstacle that to many physicists had seemed to be inachievable and irrelevant. Another essential idea was the fact that the developing theory was always formulated as an action principle.

This ought to tell us something valuable, as can be demonstrated by a supremely important counterexample. Perhaps as much as 200 years ago, and as recently as 70 years ago, there were leading scientists who advocated the development of action principles for thermodynamics and hydrodynamics. (Helmholtz 1883, Poincare 1908, Maxwell (see Ruckeyser 1942), von Neumann 1945.) In the 1930's there arose the need for a relativistic action for extended material system to serve as a source for Einstein's General Theory of Relativity. But instead of making an all out effort to develop this concept the alternative of a much less ambitious phenomenological attitude was adopted. The result can be seen in a large compilation of papers that have little or no relevance today.

\section{The legacy of J. W. Gibbs}

Gibbs fundamental paper of 1870-1875 could have been the beginning of the development of an action principle for thermodynamics. Let us try to understand why that did not happen. Of course, Gibbs never used the words Action Principle or Lagrangian. Even Maxwell, who expressed a strong admiration for Gibbs' work, and who strongly advocated action principles, made no move in that direction. One reason for this was the choice of variables adopted by Gibbs in his approach to composite or extended systems.

For a multicomponent system, such as a mixture of gases of different kinds, Gibbs used the temperature $T$, the total volume $V$, the total entropy $S$ and the molar fractions. An alternative is to use only intensive variables, the individual densities, the individual specific entropy densities and the temperature. The advantages are considerable:

There is no entropy of mixing and no free energy of mixing, no Gibbs paradox and no Gibbs-Duhem relation, no use for control volumes and no need for a separate study of open systems. But that is only the beginning.

One of the most important aspects of Gibbs' work is the attitude taken with respect to entropy. For a unary system $S$ is a parameter that is to be held fixed, along with the pressure, as the temperature and the volume are varied. As we pass from global systems actually invoked by Gibbs, to local systems characterized by field variables, we need to decide how to localize the expression $F+S T$. for a unary system the usual practice is to replace

$$
F(V, T) \rightarrow \int d^{3} x \rho f(\rho, T),
$$

where $f$ is the free energy density and

$$
S \rightarrow \int d^{3} x \rho S
$$


where from now on $S$ is the specific entropy density, almost always taken to be uniform. Indeed, that is implied by the use of the hydrostatic relation or the Bernoulli equation in the hydrodynamical context. The only circumstances in which the specific density is not (piecewise) uniform are those that involve energy consumption, such as explosions.

In this context, the entropy distribution is a quantity to which is assigned a uniform value. Circumstances in which this is not appropriate exist but they are exceptional, which is why entropic forces are rarely evoked in applications of hydrodynamics.

\section{Local thermodynamic potentials}

Localization, the passage from global thermodynamics to continuum thermodynamics, puts a new face on the theory of thermodynamic potentials. What is discussed here will become important later on, when the set of dynamical variables is expanded to include several densities.

The global theory deals with the following quartet

$\begin{array}{ccc}U(V, S) & S T & F(V, T) \\ V P & & V P \\ H(P, S) & S T & G(P, T)\end{array}$

of potentials and associated Legendre transformations. The letters $S T$ between the potentials $U$ and $F$ reminds us of the substitution $S \mapsto T$ in the Legendre transformation. The potentials that have appeared so far refer to a fixed quantity of fluid, a fixed total mass as one gram or one mole. To convert them to densities we multiply each by the mass density, $\rho U=u, \rho F=f$ and so on. In addition, we change variables, setting $V=1 / \rho$ and $S=s / \rho$. Then we find that

$$
f(\rho, T)=u(\rho, s)-s T, \quad g(p, T)=h(p, s)-s T, \quad s=-\left.\frac{\partial f}{\partial T}\right|_{\rho}=-\left.\frac{\partial g}{\partial T}\right|_{p}
$$

and

$$
g(p, T)=f+p, \quad h(p, s)=u+p, \quad p=\rho \frac{\partial f}{\partial \rho}-f, \quad \hat{p}=u-\rho \frac{\partial u}{\partial \rho} .
$$

The first line brings no surprise but the second line informs us that the pressure $p(T, \mu$ has become a potential. Instead of the quartet of global potential energies we now have a quartet of potential densities,

$$
\begin{array}{rll}
\hat{p}(s, \mu) & s T & p(T, \mu) \\
\rho \mu & & \rho \mu \\
u(s, \rho) & s T & f(T, \rho)
\end{array}
$$

where

$$
\mu:=\left.\frac{\partial f}{\partial \rho}\right|_{T}=\left.\frac{\partial u}{\partial \rho}\right|_{s}
$$


is the chemical potential. It will play an important role in the theory. The natural variables for $p$ are $T$ and $\mu$. Among the partial derivatives there is

$$
\left.\frac{\partial p}{\partial T}\right|_{\mu}=-s
$$

known as the Clapeyron equation.

We shall rely principally on the density potentials $u, s$ and $f$, functions of the density, the entropy density and the temperature. The total number of particles will not be employed as a variable and there will be no distinction between open and closed systems.

In terms of densities the main relations of thermodynamics take the form

$$
\left.\frac{\partial}{\partial T}(f+s T)\right|_{\rho, S}=0, \quad \dot{\Phi}-\vec{v}^{2} / 2-\left.\frac{\partial}{\partial \rho}(f+s T)\right|_{T, S}=\text { constant }
$$

or

$$
\left.\frac{\partial f}{\partial T}\right|_{\rho}+s=0, \quad \dot{\Phi}-\vec{v}^{2} / 2-\mu=\text { constant }
$$

\section{The Hamiltonian of thermodynamics}

This section has no foreseeable practical value. The issue is to justify the identification of the free energy as the Hamiltonian, which is usually taken for granted. But when we ask for a justification we are led to some speculations about the interpretation of the entropy that may lead to a useful debate.

We are ready to resume the study of the general structure of thermodynamics, including the central and most difficult issue: maximalization of the entropy.

We have an action, a Lagrangian density, a Hamiltonian density defined by the usual Legendre transformation,

$$
h=\dot{\Phi} \frac{\partial}{\partial \dot{\Phi}} \mathcal{L}-\mathcal{L}
$$

and Euler-Lagrange equations that summarize both hydrodynamics and thermodynamics. What we do not have so far is a principle of minimum energy.

\section{The classical Lagrangian paradigm}

So far, the action principle provides all the laws of traditional thermodynamics, including some that go beyond the description of the states of equilibrium. What is missing is part of the interpretation. To complete the picture in this respect we can do no better than to seek inspiration from tradition. We need to understand how it comes about that the Hamiltonian of an action principle tends to have the lowest value at equilibrium. The paradigm of an action principle is one that involves dynamical variables $q_{1}, \ldots, q_{n}$ and

$$
\mathcal{L}=\sum \dot{q}_{i}^{2} / 2-V\left(q_{1}, \ldots, q_{n}\right)=\sum \dot{q}_{i} p_{i}-H\left(q_{1}, . ., q_{n}, p_{1}, . ., p_{n}\right) .
$$

This system is non degenerate, each variable has an independent conjugate momentum,

$$
p_{i}=\frac{\partial \mathcal{L}}{\partial \dot{q}_{i}}
$$


The equations of motion in Hamiltonian form are

$$
\dot{q}_{i}=\frac{\partial H}{\partial p_{i}}, \quad \dot{p}_{i}=-\frac{\partial H}{\partial q_{i}}, \quad i=1, \ldots, n .
$$

Here we see what we are looking for: If "equilibrium" is defined as a solution in which nothing varies with the time, $p_{1}, \ldots, p_{n}, q_{1}, \ldots, q_{n}$ being time independent, then Eq.(.2) tells us that the Hamiltonian must be stationary at equilibrium.

Turning to our action principle for hydrodynamics, with its one-dimensional canonical structure, we would define the Hamiltonian by a Legendre transformation

$$
\int d^{3} x \mathcal{L}=\int d^{3} x \rho \dot{\Phi}-H,
$$

thus $H=F+S T=\int d^{3} x(f+s T)$. This is a close, field theoretic analogue of Lagrangian particle mechanics, and the conclusion is the same: At equilibrium the Hamiltonian $H$ is extremal (assuming the appropriate definition of the Lagrangian multiplier included in $\dot{\Phi}$.$) .$

However, it has become established that the 'energy' functional that takes a minimal value in many applications of thermodynamics to an isolated system (with fixed total volume) is the free energy

$$
F=\int d^{3} x f(\rho, T)
$$

The 'energy' density is the free energy density $f$. The Hamiltonian density $h$, on the other hand as defined by (7.3) or

$$
\mathcal{L}=\rho \dot{\Phi}-h
$$

is $h=\rho \vec{v}^{2} / 2+f+s T$. The problem is that the 'energy' of classical, equilibrium thermodynamics is not the Hamiltonian of the Action Principle. A solution is to regard the term $s T$ as part of the kinetic energy density, ${ }^{*}$ by interpreting $S$ as a time derivative.

This should tell us something about the physical interpretation of entropy. The value of the entropy can be changed by an interaction with the environment. It is a record of past interactions between our system and the environment, an interaction that is no longer active. That is why neither temperature nor entropy appears in the Lagrangian as a time derivative. We shall have to generalize this situation, and we must try to do it without altering the essential properties of the theory.

Consider replacing, in the Lagrangian density, the term $s T$ :

$$
s T \rightarrow T \rho \dot{\sigma}+j(\sigma)
$$

We have introduced a new field variable $\sigma$ and a source density $j$. Variation of $T$ gives

$$
\frac{\partial f}{\partial T}+\rho \dot{\sigma}=0
$$

* The "kinetic part" of the Lagrangian density is the part that contains time derivatives. 
which tells us that $\dot{\sigma}$ is the specific entropy density. Variation of the mass density gives the familiar equation of motion with $S$ renamed $\dot{\sigma}$. We have one additional equation, from variation of $\sigma$,

$$
\frac{d}{d t}(\rho T)=\frac{\delta j}{\delta \sigma}
$$

The source $j(\sigma)$ is for us to choose and we shall take advantage of that by demanding that the systems decouple. That is, we interpret the last equation as a constraint on the right side, with no implications for the left side. The external part of the enlarged system is chosen to allows the original system to obey its own equations of motion. This is reasonable, for the only thing that we know and want to know about the extension of the system by the new degree of freedom represented by $\sigma$ is that it should maintain and justify the assumptions that were made about the 'isolated' system.

After the replacement (7.4) we have two canonical pairs of dynamical variables and a non-degenerate symplectic structure. The Hamiltonian density is now defined by - instead of $(7.3)$ :

$$
\mathcal{L}=\rho \dot{\Phi}-\rho T \dot{\sigma}-h, \quad h=\rho \vec{v}^{2} / 2+f+j(\sigma) .
$$

The Hamiltonian is extremal for allowed variations of the equilibrium state, variations that do not affect the field $S=\dot{\sigma}$. Finally, the functional $\int d^{3} x\left(\rho \vec{v}^{2} / 2+f\right)$ is extremal with respect to variations that do not affect the external source $j(\sigma)$. The term $j(\sigma)$ is inconsequential and will be dropped from now on. It may be resurrected when needed, as when there is a flow of heat in or out of the system.

\section{Summary}

Prompted by the experimental evidence we have moved the term $s T$ out of the Hamiltonian. The field $\sigma$ is canonically conjugate to $\rho T$, the canonical structure is non degenerate.

We have developed the interpretation of the entropy in a direction that helps us get a better grasp on its nature. The parameter $S$ acts like a Lagrangian multiplier, in that the actual value is determined by a one-sided influence of the environment. The system approaches an equilibrium state by giving up energy to the environment while it remains constrained by the principle of least action; this necessarily leads to adopting the highest value of the total entropy. The environment has infinite entropy and is not affected in a measurable way; just as the reflection of a small mass from a very large one has no measurable effect on the latter because the momentum change is measurable only through observation of the velocity change $\Delta v=M^{-1} \Delta p$.

These conclusions are not the result of speculation but are based on the experimental fact that it is the free energy, rather than the internal energy, that is minimal for equilibrium configurations of an isolated system.

\section{The Lagrangian method}

\section{A theory of Mixtures}

The traditional treatment of mixtures often relies on rules concerning the additivity of properties; one of the most important ones is the additivity of the energies of the 
components of a composite system. Most fundamental of all is the postulate of additivity of Lagrangians;

$$
\mathcal{L}=\sum_{i=1,2} \mathcal{L}_{i}=\sum_{i} \rho_{i}\left(\dot{\Phi}_{i}-\vec{v}_{i}^{2} / 2\right)-f-s T,
$$

it incorporates the additivity of energies and the additivity of entropies. The algebraic sum of the Lagrangians of two adiabatic systems describes an adiabatic system with two non interacting components.

The reason why this is an unfamiliar concept is that the Lagrangian density of hydrodynamics or thermodynamics has not been given any importance till now.

Realistically, coexistent systems are never non interacting and ways have to be found to take the interaction into account. The strategy will be to begin by adding together the Lagrangians of components, with the expectation that corrective terms, 'interactions', will have to be added. The systematic application of this point of view is not wide spread in thermodynamics. (See for example many studies of critical phenomena in mixtures.) But it is a standard, and successful, strategy in more developed branches of theoretical physics.

Recall that in unary thermodynamics it is axiomatic that the free energy (or any other fundamental potential expressed in terms of the natural variables) tells us all that we need to know about a substance. It follows that a mixture in which each constituent behaves as if it were alone is described by variables that satisfy the usual equations of motion of a unary system. The natural variables for the mixture is thus the collection of natural variables for the ingredients. And if this mixture is characterized by an action principle, then the total action must be a sum of individual actions, in the limit of no interactions.

The persistent use of a Lagrangian does much to distinguish our approach to thermodynamics, the choice of variables is just as important. I confess to being perplexed by the common usage, especially the occasional suppression of degrees of freedom and the preference for molar fractions over partial densities. Here is the addition formula for free energy of a mixture of ideal gases,

$$
F=\frac{f_{1}+f_{2}}{\rho}=N_{1} F_{1}+N_{2} F_{2}+\mathcal{R} T\left(N_{1} \ln N_{1}+N_{2} \ln N_{2}\right)
$$

In terms of ordinary densities this statement takes the form $f=f_{1}+f_{2}$.

It will be assumed that no chemical reactions are taking place in the mixtures under consideration, and that the equilibria are uniform. In this case an important idea is the Gibbs-Dalton hypothesis.

\section{Physical variables}

A description of two non interacting systems requires two sets of variables. In particular, each system has a temperature or a temperature field. This is natural if the two systems occupy different, non overlapping regions of space. Situations exist where two systems occupy the same space and for which it has nevertheless been found useful to 
maintain a notion of separate temperatures. * But here the temperature shall always be taken to be a unique field extended over the total vessel.

Taken for granted is the idea that each distinct component has an independent flow vector field. Otherwise, there would be no way to formulate the separate conservation of masses and separate equations of continuity, for each component (and no way to visualise the 'mixing'). More precisely, we say that, if two densities are separately conserved, then one needs two independent velocity fields $\vec{v}_{1}$ and $\vec{v}_{2}$ in order to be able to assert that $\dot{\rho}_{1}+\operatorname{div}\left(\rho_{1} \vec{v}_{1}\right)=0$ and $\dot{\rho}_{2}+\operatorname{div}\left(\rho_{2} \vec{v}_{2}\right)=0$. ${ }^{* *}$ But if only the total density is conserved then there is only one flow and only one continuity equation. The analysis becomes simpler if one assumes that the mixture behaves as a one component fluid, ${ }^{* * *}$ with properties defined by interpolation between those of the components. This drastic assumption, first used by van der Waals, is tempting in the case of mixtures of many components; it is common, for example, in calculations relating to the interior of the Sun. But it ignores physical degrees of freedom and cannot account for all observed phenomena. And it reduces the "theory" to an exercise in phenomenology.

By adopting a two-velocity approach to all binary mixtures without chemical reactions we depart in an essential way from what is common practice. The most important 2-fluid theory is Landau's theory of superfluidity. But Landau's equations are not derived from an action principle and consequently, even in that case, we shall take a new direction.

In the ideal case, when there is no interaction between the components, there can be no objection to two separate and independent velocities; they are unaware of each other and they pass freely through each other. But this transparency will be much affected when we allow for an interaction between the components, the simplest being an addition to the total free energy density,

$$
f=f_{1}+f_{2}+f_{\text {int }}, \quad f_{\text {int }}=\alpha \rho_{1} \rho_{2}, \quad \alpha \text { constant }
$$

An interaction of this type was used with some success by van der Waals (!873). With $\alpha$ positive it has the effect of discouraging the overlap of the densities of the two species.

From now on it is taken for granted that the dynamical variables of mixtures at rest are the individual densities $\rho_{i}$, individual specific entropies $S_{i}$ (next section), and $T$. The variables chosen by Gibbs, and used almost universally ever since, are the total volume and the molar fractions, instead of the individual densities.

\section{The Gibbs-Dalton hypothesis for ideal gases}

The additivity of energies that is implied by the addition of Lagrangians is an attractive assumption, and probably a must in the absence of interactions, but what can be

\footnotetext{
* For example, in Plasma Physics, and in Cosmology.

** As in Landau's 2-fluid theory of liquid helium with its 2 equations of continuity. *** "Air is a mixture that behaves, under normal conditions, like an ideal gas." Mueller 2009.
} 
done about the two entropy parameters? Addition of heat to the system must change the parameters and if the system is to end up in a well defined configuration then it has to follow a fixed path in the 'entropy plane' the Euklidean plane with coordinates $S_{1}, S_{2}$. A partial answer to this question is provided by a literal interpretation of the Gibbs-Dalton hypothesis, developed by Gibbs from Dalton's law, namely

Gibbs-Dalton hypothesis, weak form. The entropy of a mixture of ideal gases is additive in the same sense that the free energy density is additive.

That is,

$$
s=\sum s_{i}=\sum \rho_{i} S_{i},
$$

with uniform values of $S_{1}$ and $S_{2}$; that is, this is the correct expression to be used for the function $s\left(\rho_{1}, \rho_{2}\right)$ in Eq.(8.1).

A stronger interpretation of the Gibbs - Dalton hypothesis is possible. In addition to (9.1) it assumes that

\section{When the mixture of ideal gases reaches a final equilibrium each gas has the entropy that it would have if it were alone at the same density and temperature.}

When each gas is alone these relations hold:

$$
S_{i}=-\mathcal{R}_{i}\left(\ln \frac{\rho_{i}}{T^{n_{i}}}-n_{i}\right), \quad i=1,2 \quad \text { separated gases. }
$$

According to the strong version of the hypothesis it holds for a mixture, but only at equilibrium.

$$
S_{i}=-\mathcal{R}_{i}\left(\ln \frac{\rho_{i}}{T^{n_{i}}}-n_{i}\right), \quad i=1,2 \quad \text { at equilibrium. }
$$

This is what we are going to mean by 'the strong Gibbs-Dalton hypothesis' (for an ideal gas). It is expected to holdfor miscible gases in the absence of any chemical reactions. The virtue of this statement is that, as a statement about the value of the entropy, it applies to the adiabatic system in general, not just to equilibrium states; it removes the ambiguity in the Lagrangian by fixing a relation between the two entropy parameters defined in (9.1).

Consider two ideal gases with

$$
f_{i}+s_{i} T=\mathcal{R}_{i} \rho_{i} T \ln \frac{\rho_{i}}{T^{n_{i}}}+\rho_{i} S_{i} T, \quad i=1,2 .
$$

If each gas were alone we should have the 2 equations of motion (adiabatic relations),

$$
\mathcal{R}_{i} \rho_{i}\left(\ln \frac{\rho_{i}}{T^{n_{i}}}-n_{i}\right)+\rho_{i} S_{i}=0, \quad i=1,2,
$$

from variation of each Lagrangian with respect to the temperature, but variation of the proposed total Lagrangian (9.1) with respect to the common temperature yields only one adiabatic equation: *

$$
\sum_{i=1,2}\left(\mathcal{R}_{i} \rho_{i}\left(\ln \frac{\rho_{i}}{T^{n_{i}}}-n_{i}\right)+\rho_{i} S_{i}\right)=0
$$

* This is enough to give the formula for the internal energy density, $u=\sum_{i} n_{i} \mathcal{R}_{i} \rho_{i} T$. 
The strong Gibbs-Dalton hypothesis requires that both of Eq.s (9.2) hold for the unified system, but only at equilibrium. If the equilibrium is characterized by uniform densities then this amounts to the required relation between $S_{1}$ and $S_{2}$, a relation that provides the required path in the entropy plane. Suppose that there is a value $T_{0}$ of $T$ such that both terms vanish, then for all $T$

$$
\mathcal{R}_{i} \rho_{i}\left(\ln \frac{\rho_{i}}{T^{n_{i}}}-n_{i}\right)-\rho_{i} S_{i}=n_{i} \mathcal{R}_{i} \rho_{i} \ln \frac{T_{0}}{T}
$$

and the equation of motion Eq.(9.4) reduces to

$$
\left(\sum n_{i} R_{i} \rho_{i}\right) \ln \frac{T_{0}}{T}=0
$$

This can be true only at $T=T_{0}$. That is, if such a value of $T$ exists when the densities take equilibrium values, then the equation of motion implies that this is the value of $T$ at equilibrium and that both terms vanish separately at equilibrium.

Away from equilibrium only (9.4) holds and each component gas no longer behaves as if the other component were absent! The two gases at equilibrium may not be 'aware of each other', but in configurations other than equilibrium the pair of adiabatic conditions that characterize the pure gases is replaced by the single adiabatic condition (9.2).

For a system of ideal gases with fixed total quantities of each component and with uniform equilibrium densities the Gibbs-Dalton hypothesis predicts the following entropy path,

$$
\frac{S_{1}}{n_{1} \mathcal{R}_{1}}-\frac{S_{2}}{n_{2} \mathcal{R}_{2}}=\ln \frac{\rho_{2}^{1 / n_{2}}}{\rho_{1}^{1 / n_{1}}}
$$

where $\rho_{1}, \rho_{2}$ are the densities at equilibrium. It agrees with Boltzmann's distribution law if $n_{1}=n_{2}$. 


\section{... and what lies behind it}

We have attempted to determine what it is that would drive the mixed gas to satisfy the strong version of the Gibbs-Dalton hypotheses. * Let

$$
x=\frac{\rho_{1}}{k_{01}(\mathrm{eT})^{\mathrm{n}_{1}}}, \quad y=\frac{\rho_{2}}{k_{02}(\mathrm{eT})^{\mathrm{n}_{2}}}, \quad S_{i}=-\mathcal{R}_{i} \ln k_{0 i}, \quad i=1,2,
$$

so that the Gibbs-Dalton hypothesis makes $x=y=1$, and the adiabatic relation (9.4) takes the form

$$
x \ln x+a y \ln y=0, \quad a=\left(\mathcal{R}_{2} k_{02} / \mathcal{R}_{1} k_{01}\right)(\mathrm{eT})^{\mathrm{n}_{2}-\mathrm{n}_{1}} .
$$

Let us fix $S_{1}$ and $S_{2}$, and consider an equilibrium configuration in which all the fields are uniform. Fig. 2 shows the locus of points in the $x, y$ plane at which Eq.(9.4) holds, for a particular value of $T$. Since the variables $x, y$ are constant multiples of the densities these variables are in fact scaled densities and we may refer to the plane of the figure as the density plane. There is a family of such curves, one for each value of $T$. The figure also shows several lines of constant total pressure,

$$
p=\left(\rho_{1} \frac{\partial}{\partial \rho_{1}}+\rho_{2} \frac{\partial}{\partial \rho_{2}}-1\right)\left(f_{1}+f_{2}\right)=T\left(\mathcal{R}_{1} \rho_{1}+\mathcal{R}_{2} \rho_{2}\right) .
$$

The loci of $P$ are straight lines. The isotherm intersects the isobar at 2 points, at one point, or at no point in the $\rho$-plane. If they intersect at no point then there is no such configuration.

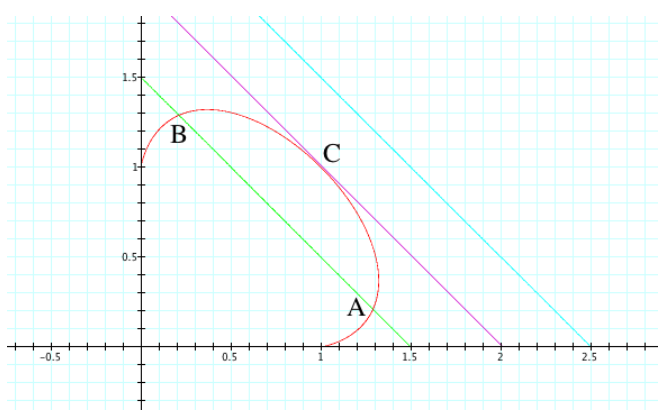

Fig. 2. The strong Gibbs - Dalton hypothesis.

If the curves touch at one point then the densities are uniquely determined. The tangents of the two curves at any point are given by

$$
\mathcal{R}_{1} d \rho_{1}(\ln x+1)+\mathcal{R}_{2} d \rho_{2}(\ln y+1)=0
$$

and

$$
\mathcal{R}_{1} d \rho_{1}+\mathcal{R}_{2} d \rho_{2}+\left(\mathcal{R}_{1} \rho_{1}+\mathcal{R}_{2} \rho_{2}\right) \frac{d T}{T}=0
$$

\footnotetext{
* The answer given by Boltzmann to this question is the foundation of statistical mechanics. Here we are looking for an answer within our chosen context of thermodynamic field theory.
} 
respectively; they coincide, for a fixed value of $T$, only if

$$
\ln x=\ln y .
$$

By virtue of Eq. (9.6) that implies that both logarithms are zero, as required by the Gibbs-Dalton hypothesis.

There remains the possibility of two intersections, at points $A$ and $B$ in the figure. To justify the Gibbs-Dalton hypothesis we have to throw some aspersions on this case. Well, if the gas at equilibrium is homogeneous, then the ratio $r=\rho_{1} / \rho_{2}$ is given in advance by the ratio of total masses. This number need not coincide with the value of either of the ratios $r(A)$ or $r(B)$. If it is intermediate between those values then the gas must separate into two parts, one part in which $r=r(A)$ and another part in which it is $r=r(B)$. Mixtures exist, where this does indeed happen, but in the case of ideal gases, or at high temperature or low pressure for most gases, we know that it does not; so there is no stationary state available. That is the same as to say that such states are adiabatically unstable to convection. Flow will develop and eventually the kinetic energy will degenerate to heat. The final state will be stationary but the values of the entropy parameters must have changed.

The only stable, homogeneous equilibrium available is the point $C$ where the two curves have the same tangent. At that point both terms in (9.4) vanish separately, as we have shown.

If the values of the densities and of the temperature are given, then this is a restriction on the values of the two entropy parameters $S_{1}$ and $S_{2}$. For a given system with fixed mass and with uniform equilibrium densities, there will be a curve $T \mapsto\left(S_{1}(T), S_{2}(T)\right)$, the image of which is the path followed by the system as the temperature is varied so as to move it through a succession of equilibrium configurations.

We seem to be able to conclude that the Gibbs-Dalton hypothesis finds the only stable mixture and that this is what determines how the entropy is apportioned between the two components. An argument based on the maximal entropy principle was expected. Instead we have here an analogue of free expansion; the assumed initial point is unstable and goes into an agitated state, out of equilibrium. The final equilibrium is reached by degradation of the kinetic energy, leading to a state of maximal entropy.

Thus it seems that the Gibbs-Dalton distribution of entropy is not reached by a purely dissipative process but because it is the only one that allows for the existence of equilibrium states, or more generally for any stable adiabatic system.

It seems that the entropy chosen by the Gibbs-Dalton hypothesis is a kind of critical point. Recall that, in general, a critical point in thermodynamics is one in which a Legendre transformation is singular (Tisza 1970). For example, consider the Legendre transformation

$$
p\left(T, q_{1}, q_{2}\right)=\sum \rho_{i} \mu_{i}-f, \quad \mu_{i}=\frac{\partial f}{\partial \rho_{i}}, \quad i=1,2 .
$$

An example of a critical point is a point where

$$
\operatorname{det}\left(\partial^{2} p / \partial \rho_{i} \partial \rho_{j}\right)=0
$$


The critical point that is identified by the Gibbs-Dalton hypothesis is defined by the vanishing of the Jacobean of the transformation $T, p \rightarrow \rho_{1}, \rho_{2}$,

$$
J=\left|\frac{\partial T \partial p}{\partial \rho_{i} \partial \rho_{j}}\right|
$$

that is, a point where the two gradients in the density plane

$$
\left(\frac{\partial T}{\partial \rho_{1}}, \frac{\partial T}{\partial \rho_{2}}\right), \quad\left(\frac{\partial p}{\partial \rho_{1}}, \frac{\partial p}{\partial \rho_{2}}\right)
$$

are parallel. The derivations are to be taken with $S_{1}, S_{2}$ fixed.

With this interpretation we can ask for a generalization of the hypothesis to the case of a mixture of real, interacting gases. In particular, it may be that this hypothesis applies to the altitude variation of the concentration of Nitrogen and Oxygen in the terrestrial atmosphere.

The suggested form of the Lagrangian for a mixture, with the concept of an entropy space and the need to find the path through this space chosen by the system, is a central feature of the treatment proposed in my book (Fronsdal 2019).

The calculation of the speed of sound in a mixture of gases needs to know the partition of the entropy for the equilibrium state. The Gibbs-Dalton hypothesis was used for this purpose in a calculation of the speed of sound in some mixtures. (Fronsdal 2019).

\section{0. introduction}

\section{Why Loschmidt went to Canossa}

The battle that Loschmidt launched, and lost, is one of these disagreements that arise from diffferent points of view. With statistical mechanics Boltzmann and Maxwell built a formidable and in all aspects successful theory of statistical mechanics. As that theory has remained a theory of equilibria it has been well served by a popular dictum: The temperature of an extended system in equilibrium is uniform. In thermodynamic this is associated with the second law, the axiom of largest entropy.

Let us try to make the case from our point of view. The laws of thermodynamics are the Eeuler- Lagrange equations of an action that depends parametrically on $P$ and on $S$. For an extended system $P$ is replaced by a surface field and the entropy by an entropy density.

Gibbs' paper has a long discussion, over several pages, about the choice of the space of variations. Then, on subsequent pages he includes, in the space of variations, local variations of entropy. Gibbs endows entropy with the property of being subject to interchange between two regions, an increase in one with an equal, compensatory decrease in the other. As was emphatically emphasized by Prigogine (1949), and as we teach our students, such compensation takes place only when the temperatures are equal, since

$$
d Q=T_{1} \Delta S_{1}+T_{2} \Delta S_{2}=0
$$


Once an action principle has been adopted, with a well defined space of variations, one can of course ask if there is merit in enlarging that space. But beware that this will lead to additional Euler-Lagrange equations that will be violated by some solutions of the original action principle. A pragmatic approach is indicated, and this attitude has dominated the history of applications of thermodynamics. In the present instance it consists of eschewing, as a working hypothesis, local variations of the entropy. With this attitude a theory of atmospheres has been developed, notably for stellar and planetary atmospheres. In astrophysics the main object of analysis, since the beginning, has been static configurations with extreme and universally recognized temperature variations.

Consider a laboratory vessel filled with an ideal gas, neglecting the effect of gravity. In a hydrodynamic treatment the temperature is usually taken to be constant. The equations of motion are the equation of continuity and the hydrostatic equation. When no external forces and no entropic forces are acting it follows from these equations that, in the static case, the pressure and the density are uniform. The equations used are the Euler-Lagrange equations of the action

$$
A=\int d^{3} x\left(\rho\left(\dot{\Phi}-\vec{v}^{2} / 2\right)-W[\rho]\right)
$$

that was discovered by Lagrange ca 1760 . Here $\Phi$ is the velocity potential, $\vec{v}=-\vec{\nabla} \Phi$.

The effect of gravity is surely known in this case, one simply modifies the Lagrangian density by $-\rho \phi$, where $\varphi$ is the Newtonian potential $g z$. In case of doubt it can be verified that this prescription is consistent with the Einstein's metric theory of gravity (Fronsdal 2007). The effect is to make $\rho$ and $p$ vary with the altitude. This result implies that the temperature also varies with altitude, which is inconsistent with our initial assumption. Standard hydrodynamics is not the answer.

Turning back to thermodynamics we must make an assumption about the entropy. The great advantage of a uniform specific entropy is that, in this case only there are no entropic forces and the usual hydrostatic condition of hydrodynamics is validated. Choosing any other particular $S(z)$ fails to give a uniform temperature. It has been suggested to postulate

$$
s=\rho S+s_{e x}(z)
$$

but this is inconsistent with the equations of localized thermodynamics.

In short, the dynamical approach based on an action principle is an excellent example of the superior predictive power of action principles.

We want to know what is different when gravity is taken into account; especially, if the equilibrium temperature is still uniform. In hydrodynamics the temperature, not necessarily uniform, it is eliminated by using the adiabatic condition

$$
S=-\frac{\partial f}{\partial T}
$$

which gives no information unless the specific entropy field is known. If $S$ and $T$ are both uniform, as in the absence of an external field, then $\rho$ and $p$ are uniform as well; in the presence of gravity this is inconsistent with the hydrostatic condition. 
Consider an atmosphere that consists of an ideal, static gas with the action (10.1) with the usual addition that represents the gravitational field,

$$
A=\int d^{3} x\left(\rho\left(\dot{\Phi}-\vec{v}^{2} / 2-\varphi\right)-W[\rho]\right)
$$

where $\varphi$ is the terrestrial, gravitational potential, $\varphi=g z$ and

$$
f(\rho, T)=\mathcal{R} \rho \ln \frac{\rho}{T^{n}}
$$

The two Euler-Lagrange equations, combined so as to eliminate the logarithm, yields

$$
g z+\mathcal{R}(n+1) T=\text { constant }
$$

the famous temperature lapse, as shown in all the textbooks. This result is in good numerical agreement with measurements in a dry atmosphere. The derivation, and the resulting variation of $T$ with the altitude, has been accepted by astrophysicists since its inception in the 19 'th century.

It will always be objected that stars are not in equilibrium. As well explained by Eddington, the evolution of stars is characterized by (at least) two time scales. Visible, global evolution is very slow, on a short time scale, the approximation in terms of equilibria is a good one. Evolution is seen as a sequence of equilibria; to understand and to describe them we must treat them as such.

Among the textbooks on thermodynamics, from the 1950's onwards, there are some that derive the observed temperature of our atmosphere from the equations of motion, as above, then report that this result is not accepted by thermodynamicists. The temperature lapse is real, but the result is 'wrong' because a system with a variable temperature cannot be in equilibrium. The action principle of Lagrange and Laplace is not recognized; that is a strong position to take by physicists whose domain of expertize is often limited to equilibria!

More recently, textbooks on planetary atmospheres eskew a discussion of the controversy to present a straightforward, theoretical discussion, leading to the result (10.3), directly compared to measurements.

Actually, there is no real conflict but a question of the most rational approach o a complicated situation. The dyed in the wool statistical mechanician recomends taking the isothermal atmosphere, far from the actual atmosphere that is the product of convection and radiation as as a zeroth approximation. From a practical point of view this is very difficult program.

The alternative is to develop a simple model, as above, that is favored by the right temperature lapse, in agreement with experiment, to be left with the simpler task of showing that the joint effects of convection and radiation actually tend to be minor, or that they cancel out. A (theoretical) configuration of equilibrium in thermodynamics is not an equilibrium in the sense of statistical mechanics, a theory with smaller field of applications. 
The second law states that among available equilibrium fluctuations with given energy the system will choose the one with the highest entropy. The key word here is, as emphasized by Gibbs, 'available'. In the theory of Lagrange and Laplace the available configurations are those that satisfy the Euler-Lagrange equations.

\section{The variables of hydrodynamics}

\section{Conservative Hydrodynamics}

Traditional hydrodynamics knows two formulations, the Eulerian and the Lagrangian versions; they are not equivalent but more accurately described as complimentary. In the much studied case of cylindrical Couette flow two types of flow appear, usually combined in the basic velocity field of the Navier-Stokes equation. The general solution, for the simplest case of circular Couette flow is, in cylindrical coordinates,

$$
\vec{v}=\frac{a}{r^{2}}(-y, x, 0)+b(-y, x, 0), \quad r=\sqrt{x^{2}+y^{2}} .
$$

The first type is irrotational and satisfies the Navier- Stokes equation; in this special case that equation can be written in this form:

$$
\dot{\vec{v}}+\vec{\nabla} K_{1}=-\frac{p}{\rho}, \quad \vec{\nabla} K_{1}=(\vec{v} \cdot \vec{\nabla}) \vec{v}, \quad K_{1}:=\vec{v}^{2} / 2, .
$$

In the second case one obtains the same result, but with the kinematic potential

$$
K_{2}=-\vec{v}^{2} / 2, \quad \vec{\nabla} K_{2}=(\vec{v} \cdot \vec{\nabla}) \vec{v}
$$

This tells us that the first case can be accommodated by the 'Eulerian' action

$$
\int d^{3} x\left(\rho\left(\dot{\Phi}-\vec{\nabla} \Phi^{2}\right)-W[\rho]\right),
$$

the second by the 'Lagrangian' action

$$
\int d^{3} x\left(\rho \vec{\nabla} X^{2} / 2-W[\rho]\right) ;
$$

neither theory can accommodate both.

It also tells us that the concept of a kinematic potential is not consistent with the Navier - Stokes equation, nor is the concept of kinetic energy.

In the case that both flows are present, as is in fact the case in cylindrical Couette flow, the Navier-Stokes equation can be written in the form

$$
\dot{\vec{v}}+\vec{\nabla} K_{3}=-\frac{p}{\rho}
$$

with the kinetic potential

$$
K_{3}=\frac{a^{2}}{2 r^{2}}+2 a b \ln r-b^{2} r^{2} / 2
$$


but it is not associated with any action; $K_{3}$ cannot be expressed in terms of the sum $\vec{v}$ and neither of the two formulas for $K_{1}, K_{2}$ is valid..

This suggests that, if we are looking for an action for hydrodynamics, then it is not enough to allow for the general form of $\vec{v}$; we must regard the two terms as two independent degrees of freedom, as in the action

$$
A=\int d^{3} x\left(\left(\rho\left(\dot{\Phi}+\dot{\vec{X}}^{2} / 2+\kappa \dot{\vec{X}} \cdot \vec{\nabla} \Phi-\vec{\nabla} \Phi^{2} / 2\right) \rho-W[\rho]\right) .\right.
$$

\section{Degrees of freedom. Equations of motion and constraints}

The kinetic terms in (11.3) already appeared in a well known paper by Hall and Vinen (1956), in an expression for the energy of superfluid Helium. The gradient field was interpreted in terms of phonons. The vector field here denoted as a time derivative was not a field degree of freedom but the velocity associated with a rotation of the liquid as a whole: rotons. Perhaps it was felt that a dynamical field would bring in too many degrees of freedom. Lund and Regge (1976) used the field $\vec{X}$ to represent vorticity, and interpreted it has a classical, gauge fixed limit of the massless two-form field of the 'notoph' that had been examined by Ogievetskij and Palubarinov (1964). This field has only two degrees of freedom, one propagating mode, which brings the total number of degrees of freedom in (11.3) to 4 , which is what we need for hydrodynamics.

Here then is the long sought generalization of the hydrodynamic action known to Lagrange in 1760. It is unique in the following sense: It has the correct number of degrees of freedom and it preserves the all-important equation of continuity; this equation is the Euler - Lagrange equation that comes from variation of the velocity potential,

$$
\dot{\rho}+\vec{\nabla} \cdot(\rho \vec{v})=0, \quad \vec{v}:=\kappa \dot{\vec{X}}-\vec{\nabla} \vec{\Phi}
$$

The fact that the scalar velocity potential remains as a part of the set of dynamical field variables is therefore of the greatest importance. That is, an additional field is needed, not a generalization of the gradient velocity field. This field serves an additional important function, for it provides the only way to relate Einstein's metric field to non relativistic Newtonian gravitational hydrodynamics (Fronsdal 2007).

The Euler-Lagrange equations are, from variation of the scalar potential $\Phi$ the equation of continuity and from variation of the density the Bernoulli equation:

$$
\vec{\nabla}(\dot{\Phi}-K-\phi)=-\frac{1}{\rho} p, \quad K=-\dot{\vec{X}}^{2} / 2-\kappa \dot{\vec{X}} \cdot \vec{\nabla} \Phi+\vec{\nabla} \Phi^{2} / 2
$$

It has a strong flavor of the Navier - Stokes equation. From variation of $\vec{X}$,

$$
\frac{d}{d t}(\rho \vec{w})=0
$$


Energy conservation is lost in the presence of dissipation, but some idea concerning the effect of viscocity can be obtained by replacing the last equation by

$$
\frac{d}{d t}(\rho \vec{w})=\mu \rho \Delta \vec{v}
$$

The much studied stationary flows are characterized by

$$
\Delta \vec{v}=0 .
$$

The full gauge theory is the relativistic theory of a two form $Y$,

$$
Y_{i j}=\epsilon_{i j k} X^{k}, \quad Y_{0 i}=\eta_{i} .
$$

In the physical gauge $\eta=0$ and only the field $\vec{X}$ has a physical interpretation.

Besides the Euler - Lagrange equations there is a constraint, from the variation of the action with respect to the gauge field $\eta$,

$$
\vec{\nabla} \wedge(\rho \vec{w})=0, \quad \vec{w}:=\dot{\vec{X}}+\kappa \vec{\nabla} \Phi .
$$

This is what reduces the number of degrees of freedom; the 2-form has only one propagating mode; and this is what gives the theory its power of prediction.

\section{Interpretation}

An intuitive grasp of the meaning of the new degrees of freedom will come gradually, by applications. Here is a list of the simplest applications, all are stationary configurations.

Cylindrical Couette flow, steady, circular rotation of a fluid in the interval between two concentric cylinders, with the no - slip boundary condition. The simplest question of interest is the onset of instability of the most basic flow. What has been observed can be very well understood by making the hypothesis that instability happens at a specific value of the kinetic potential $K$. It is suggested that the breakdown of the horizontal flow will be local in the case that the cylinders are rotating in opposite directions but that it may be global in the case of co-rotation. It is predicted that the highest pressure must be found at the inner boundary and this suggests that the gradient of $K$ can be interpreted in terms of internal stress. It was found that the parameter $\kappa$ varies inversely with the compressibility of the fluid.

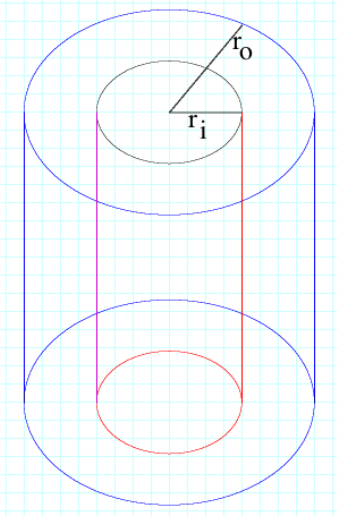

Fig. 3. Cylindrical Couette flow.

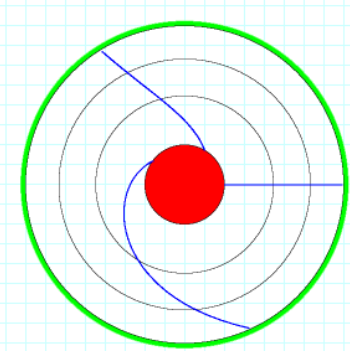

Fig.4. Traces of the flow. 
The constraint (12.3) is solved by defining a scalar field $\tau$,

$$
\rho \vec{w}=\vec{\nabla} \tau .
$$

This leads to the presentation of the mass flow velocity as

$$
\vec{v}=-\frac{\kappa}{\rho} \vec{\nabla} \tau-\left(1+\kappa^{2}\right) \vec{\nabla} \Phi .
$$

The condition $\vec{\nabla} \vec{v}=0$ is solved, in the first place, by harmonic functions of low order. Precisely the same plan was used for the other applications.

Rotating planets. The same equations were applied to planets rotating around a central axis. The density profile can be fairly fitted in the case that it is known. The parameters can be constrained by calculating the equatorial bulge. Planetary rings appear spontaneously but planetary disks have not been seen ... yet.

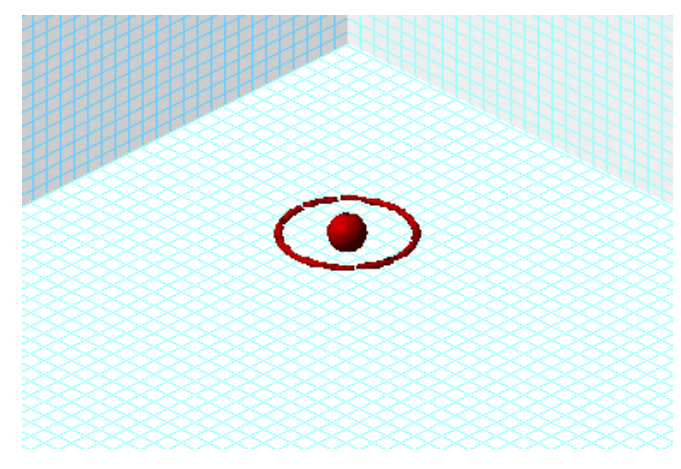

Fig. 5. A planet with ring.

The hexagonal pattern with local whorls that have been observed at the North Pole of Saturn are easy to copy in the model. Only one local whorl was seen but up to 6 are expected to greet the next traveller.

Inside the meniscus. It is traditional to explain the shape of the capillary meniscus, as well as the shape of drops and similar phenomena, in terms of a surface tension. But the curved surface of a meniscus is an isobar and the lower isobars must be curved too; hence tension is present in the bulk. The surface can be fitted to a $K$ locus and the isobars calculated throughout. Although $\vec{v}=0, \Phi$ and $\tau$ are not zero. These fields contribute to the kinetic energy and to angular momentum, and this opens fascinating possibilities for explaining certain famous experiments with rotating superfluid Helium.

Landau's original suggestion for understanding superfluid Helium employed two kinds of flow, while the idea of two kinds of $\mathrm{He}_{4}$ was explicitly rejected. (Landau 1945). Now we know that all liquids have two kinds of flow and a conservative theory of superfluids is not an unreasonable prospect. 


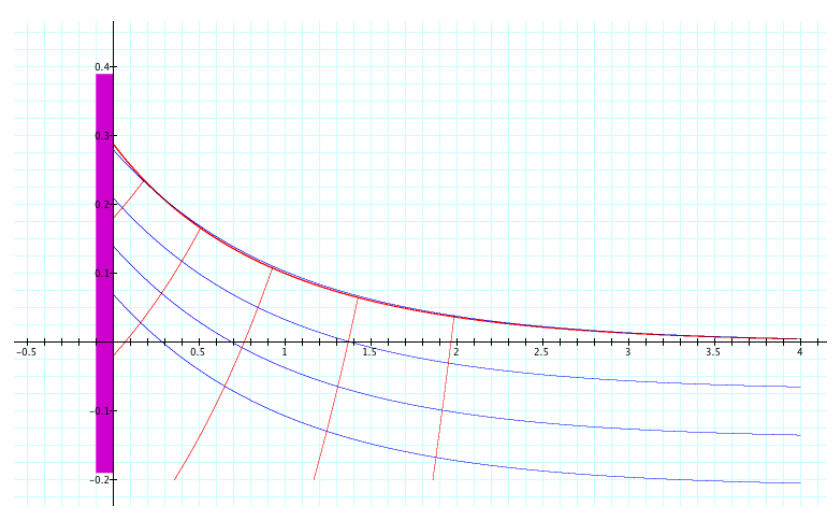

Fig. 6. The meniscus against a straight wall, with isobars under the surface.

Metastable states of fluids. Finally, this example is about thermodynamics, and entropy. Consider a piston containing a van der Waals fluid, neglecting gravity. As the piston is pulled outwards, carefully, at constant temperature, the system enters a metastable state, pressure descends, eventually to large negative values. The thermodynamic free energy can be determined from measuring the temperature and the pressure but the complete free energy has the additional, 'kinetic' contribution

$$
\int d^{3} x \frac{\rho}{2}\left(\dot{\vec{X}}^{2}+\vec{\nabla}^{2}\right)=\frac{1}{2}\left(1+\frac{1}{\kappa^{2}}\right) \int d^{3} x \vec{\nabla} \Phi^{2} .
$$

When the the system is disturbed this energy is converted to entropy, used to produce evaporation at an explosive rate ("rupture") without addition of heat.

Fig. 7. Pulling out the piston can reduce the pressure inside far below zero.

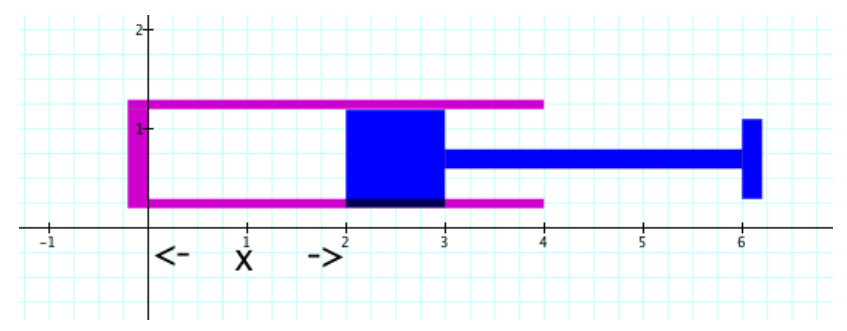

The proposed action principle for "Conservative Hydrodynamics" needs further testing. Most promising is a new attempt to account for the properties of superfluid Helium $_{4}$. Necessary generalizations include systems with several components.

Last words. The original impulse for this work was the problem of finding a continuous source for Einstein's equation, a tensor constructed from hydrodynamic and thermodynamic fields, subject to dynamical equations that imply the Bianchi identity. The work of E. Noether tells us that, given any relativistically invariant action principle constructed from tensor fields in an arbitrary background metric, there is an energy momentum tensor given in the simplest case by the formula

$$
T_{\mu}^{\nu}=\sum_{\phi} \phi_{\mu} \frac{\partial \mathcal{L}}{\partial \phi_{\nu}}-\delta_{\mu}^{\nu} \mathcal{L}
$$


or equivalently by

$$
\sqrt{-g} T^{\mu \nu}=2 \frac{\partial \sqrt{-g} \mathcal{L}}{\partial g_{\mu \nu}},
$$

where $\phi$ runs over a set of fields, $\phi_{\mu}=\partial \phi / \partial x^{\mu}$ and $\mathcal{L}$ is the Lagrangian density. This is a highly constrained program which implies a strong predictive power. The alternative, phenomenological approach is capable of accounting for observation but it has no predictive power.

Plans for the near future lie in a different direction. The new theory of fluids is likely to have applications of immense reach, theoretical and practical. Our lives are threatened by hurricanes and floods. We have learned that there may be more energy, hence more destructive power in fluids than had been known. We need to have a better understanding of the forces that control life on Earth.

Finally, if the field $\dot{\vec{X}}$ is truly ubiquitus then it will affect the motions of heavenly bodies; it will contribute to the local photon mass and effect gravitational lensing. In short, it will contribute to the effects that are usually attributed to Dark Matter.

Acknowledgements Segments of this report will appear in the book Adiabatic Thermodynamics by the same author, to be published by World Scientific in 2019. I thank World Scientific and Entropy for permission. 


\section{References}

Bernoulli, D., Argentorat, (1738).

Betchov, r. and Criminale, W.O.J, " Stability

Biot, J.B., "Sur la théorie du son", Journal de physique, 55, 173-182, 1802.Y.

Birkhoff "Hydrodynamics, Dover NY 1950.

Boltzmann, "Gastheorie", Leipzig, Barth 1896 and 1898.

Boltzmann, L., Wissenschaftlidhe Abhandlungen, Hasenoehrl, Leipzig 1909.

Boltzmann, L., Wissenschaftliche Abhandlungen, Vol.2,

Callen, H.B., Thermodynamics, John Wiley N.Y. 1960. see page 21.

Clausius, R., "Uber der mechanische Warmetheorie", Vieweg Verlag,

Braunschweig 1887, pp 93 and 357.

Clausius, R., "Uber der mechanische Warmetheorie", Vieweg Verlag,

Braunschweig 1887, pp 93 and 357.

Eddington, A.S., The internal constitution of stars, Dover, N.Y. 1959.

Fetter, A.L. and Walecka, J.D., Theoretical Mechanics of Particles and Continua, MacGraw-Hill NY 1980.

Fronsdal, C., Adiabatic Thermodynamics, to be published by World Scientific (Singapore).

A working copy is available on the author's website fronsdal.physics.ucla.edu.

Fronsdal, C. "Ideal stars in General Relativity", Gen. Rel. Grav 39, 1971-2000 (2007)

Fronsdal, C. "Heat and Gravity", Entropy (2011)

Fronsdal, C., "Action Principle for Hydrodynamics and Thermodynamics including general, rotational flows", arXiv 1405.7138v3[physics.gen-ph], (2014).

Fronsdal, C., "Relativistic Thermodynamics, a Lagrangian Field Theory for general flows including rotation", Int.J. Geom.Math.Meth. Phys., 141750017 (2017).

Fronsdal, C., "A classical mistake and what it tells us", arXive submitted (2018a).

Fronsdal, C., "Rotating planets", arXive

Fronsdal, C., Adiabatic Thermodynamics to be published by World Scientific (2019).

Gibbs, J.W., "On the equilibrium of heterogeneous substances" Trans.Conn.Acad. 1878.

Hall, H.E. and Vinen, W.F., "The Rotation of Liquid Helium II. The Theory of Mutual Friction in Uniformly Rotating Helium II", Proc. R. Soc. Lond. A 1956238 ,

Helmholtz, H. "Die Thermodynamik chemischer Vorgänte". In Wissenschaftliche

Abhandlungen; Barth, Germany, Volume II, 958-978 (1883).

H Planetary science

Kelvin, Thomson, W., Collected Mathematical and Physical papers, Vol. 5, 232-235.

Kelvin, Thomson, W., Collected Mathematical and Physical papers, Vol. 3, pp 255-260.

Lund, F. and Regge T., "Unified Approach to strings and vortices with soliton solutions", Phys. Rev. D. 14 1524-1548 (1976).

Kelvin, Thompson, W., Baltimore Lectures, C.J. Clay and Sons, London 1904.

Loschmidt, L., Ü̈ber den Zustand des Wärmegleichgewichts eines Systems von Körpern mit Rücksicht auf die Schwerkraft", Sitzungsberichte der Mathematisch-Naturwissenschaftl. Klasse der Kaiserlichen Akademie der Wissenschaften, 73.2 (1876), p. 135. 
Müller, I., A History of Thermodynamics, Springer, Berlin 2007.

Ogievetskij, V.I. and Polubarinov, I.V., "Minimal interactions between spin 0 1/2 and 1 fields", Exptl. Theoret. (U.S.S.R.) 46, 1048-1055 (March, 1964) .

Poincareé, H. Thermodynamique, Gauthier-Villars, Paris 1908.

Prigogine, I., "Evolution criteria, variational properties and fluctuations", in

"Thermodynamics, variational techniques and stability," Donnelly et al,

U. Chicago Press 1965.

Prigogine, I, Physica, 58, 272-284 (1949).

Rayleigh, L., "On the stability or instability of certain fluid motions, Proc.Lond.Math.Soc 11, 57-70 (1880).

Rukeyser, M., "Willard Gibbs, OX BOW PReSS, Woodbridge, Conn., 1942;

especially from page 199 onwards.

Thomson, W., "On an absolute Thermometric Scale, Phil. Mag. 33, 313 (1848).

Weinberg, S., "Gravitation and Cosmology: Principles and Applications of the General Theory of Relativity", John Wiley, N.Y. 1972.

van der Waals, J.D., "Over de

Doctoral thesis 1873 .

Zheltukin, A., "On brane symmetry, arXiv.1409.6655. 